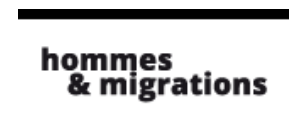

\section{Hommes \& migrations}

Revue française de référence sur les dynamiques

migratoires

Les Turcs en France : quels ancrages?

\title{
Migrations, situations familiales et relations intergénérationnelles
}

Entretien réalisé par Marie Poinsot, avec Pinar Seymen-Hüküm, Zeliha Alkis, Zeynep Acikel et Nur Gürsel

Marie Poinsot, Pinar Seymen-Hüküm, Zeliha Alkis, Zeynep Acikel et Nur Gürsel

\section{OpenEdition}

\section{Journals}

\section{Édition électronique}

URL : http://journals.openedition.org/hommesmigrations/306

DOI : 10.4000/hommesmigrations.306

ISSN : 2262-3353

Éditeur

Musée national de l'histoire de l'immigration

Édition imprimée

Date de publication : 1 juillet 2009

Pagination : 88-98

ISSN : 1142-852X

\section{Référence électronique}

Marie Poinsot, Pinar Seymen-Hüküm, Zeliha Alkis, Zeynep Acikel et Nur Gürsel, « Migrations, situations familiales et relations intergénérationnelles », Hommes \& migrations [En ligne], 1280 | 2009, mis en ligne le 29 mai 2013, consulté le 30 avril 2019. URL : http://journals.openedition.org/

hommesmigrations/306; DOI : 10.4000/hommesmigrations.306 


\section{Migrations, situations familiales et relations intergénérationnelles}

Entretien réalisé par Marie Poinsot, avec Pinar Seymen-Hüküm, responsable actions sociales - prévention

Zeliha Alkis, responsable des ateliers enfants et de la médiation scolaire

Zeynep Acikel et Nur Gürsel, permanentes sociales Association ELELE

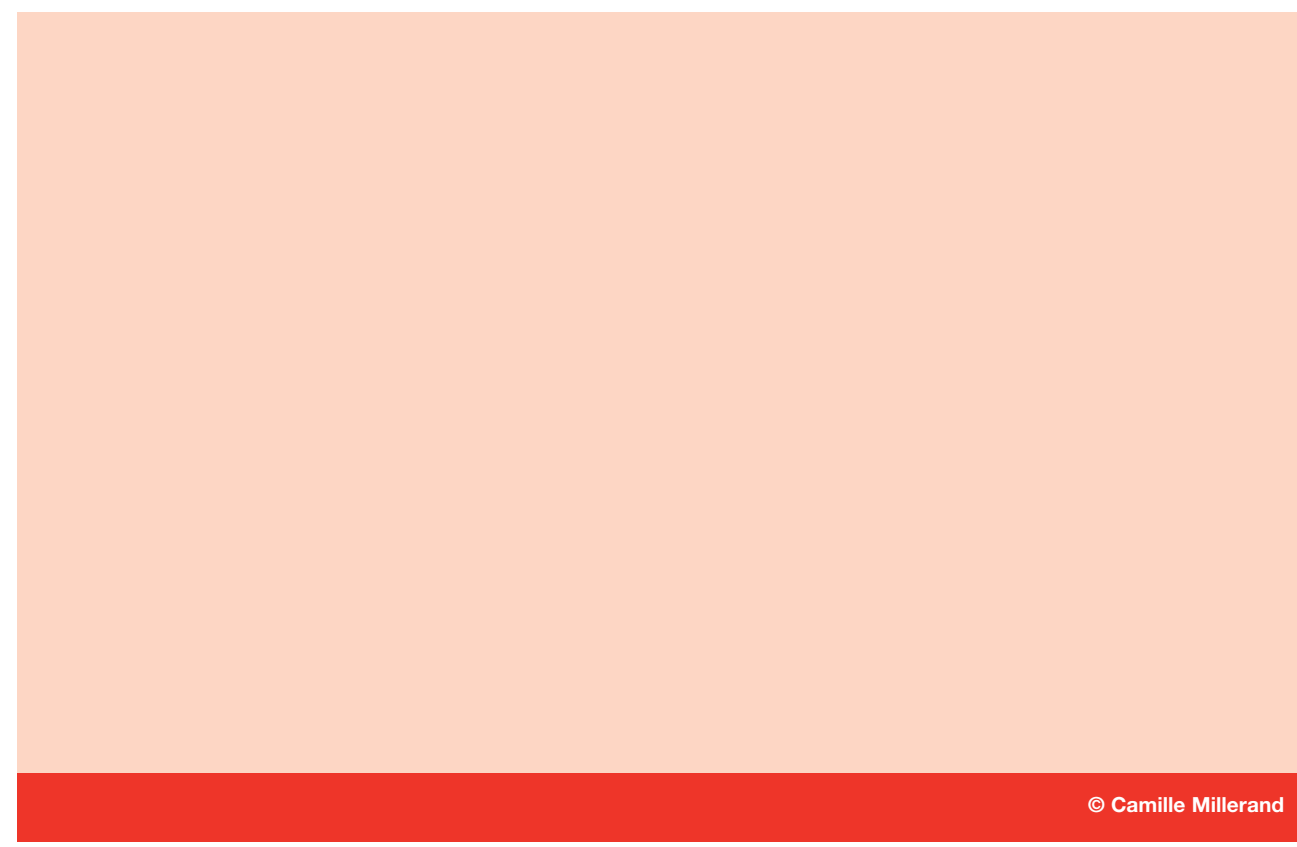

L'association ELELE - Migrations et Cultures de Turquie mène depuis 1984 des actions favorisant l'intégration en France des personnes originaires de Turquie. Créant des passerelles entre deux univers culturels, les professionnelles d'ELELE dressent un portrait lucide de la réalité sociale vécue par les immigrés turcs en France. 


\section{Quel type de public accueillez-vous chez ELELE ? Quel est son profil ?}

Au tout début, l'association ELELE accueillait surtout un public masculin, puis, petit à petit, les hommes ont pris confiance et ont laissé leurs femmes effectuer les démarches. Cela a permis aux femmes de venir nous voir, de sortir de chez elles. C'étaient dans les dix premières années d'ELELE. Maintenant, le public est surtout féminin, car nous menons des actions spécifiques dans sa direction. Cependant, tout ce qui relève des pratiques administratives et juridiques reste du ressort des hommes. Notre public est donc mixte.

Les femmes ont commencé à venir pour traiter des questions qui les intéressent tout particulièrement, des questions familiales, liées aux enfants, ou tout simplement parce qu'elles trouvent à ELELE des personnes avec qui elles peuvent communiquer plus facilement.

Une partie importante de notre public est constituée de personnes installées en France depuis longtemps. Par ailleurs, des nouveaux arrivants ou bien des conjoints sollicitent ELELE avant de faire venir leur famille, ou bien nous les recontactons après la venue du conjoint pour proposer un suivi afin de faciliter l'intégration. De fait, l'association est signalée depuis longtemps pour le traitement des questions liées à l'accueil des personnes.

\section{Quelles sont les difficultés que rencontrent les populations que vous recevez?}

Le public installé en France est confronté à des difficultés qui ne sont pas liées uniquement au problème de la langue, mais aussi aux situations sociales, au logement, à la réalité du monde actuel, au vieillissement, à l'isolement, à la précarisation de plus en plus évidente des personnes.

L'Île-de-France présente une grande concentration de population d'origine turque, y compris dans des localités ou des quartiers qui ne sont pas conçus pour accueillir des familles et dont les logements ne sont pas en adéquation avec la taille de la famille. À leur arrivée, les primo-arrivants trouvent une France des banlieues, où les bus arrivent au bout de 20 minutes, des petits appartements, des quartiers dégradés et une vie sociale réduite à la sphère communautaire. C'est un choc, qui prime sur le bonheur de rejoindre le conjoint. La France imaginée est mise à mal par la France réelle. Enfin, au choc de l'environnement ou de l'accueil français se superpose la surprise face au type de communauté dans laquelle ils arrivent. 


\section{Ces personnes anivent-elles dans le cadre du regroupement familial ou pour rejoindre un conjoint d'origine française?}

La majorité des arrivées concerne les mariages ayant eu lieu avec un conjoint venant de Turquie. Ces conjoints turcs ne viennent donc pas du même milieu éducatif que les beaux-parents, ni de la même société. Leurs normes et leurs repères ne sont pas les mêmes. Ces conjoints venus de Turquie pour rejoindre un partenaire français pensent arriver dans une famille installée en France depuis longtemps, donc évoluée, émancipée, ouverte sur la société d'accueil, tolérante. Or le premier choc est justement l'enclavement qu'ils constatent à leur arrivée.

\section{Est-ce que cela relève toujours de la pratique des mariages arrangés?}

Ce n'est pas un phénomène radicalement nouveau, mais la nouveauté réside dans la fréquence des mariages aujourd'hui. L'immigration turque commence à avoir toute une génération d'enfants nés en France ou arrivés ici en bas âge et qui parviennent à l'âge du mariage. Ce type de mariage, avec un conjoint turc, n'était pas si fréquemment pratiqué auparavant, faute de jeunes d'origine turque nés en France en âge de se marier.

Un autre facteur a modifié la donne : pour une fille éduquée en France, ayant eu son bac, on ne peut plus, comme on le faisait avant, aller lui chercher en Turquie un mari qui n'a pas fait d'études, un simple paysan. Ainsi, si l'on fait référence au niveau d'éducation des jeunes Français d'origine turque, on peut constater que leurs conjoints primo-arrivants ont un niveau d'éducation beaucoup plus élevé que celui de leurs parents arrivés de Turquie il y a longtemps. C'est donc cette différence de valeur qui joue, de même que l'image de la belle-famille dans lequel le primo-arrivant s'est projeté avant même de venir en France.

On constate que les jeunes hommes venant de Turquie ont aujourd'hui un niveau scolaire nettement supérieur à celui des conjoints vivant en France. Si, pour les garçons, il ne faut pas que la femme soit plus éduquée que le mari, pour les jeunes filles nées en France, les familles auront aujourd'hui tendance à rechercher un mari ayant un niveau social, économique et/ou d'éducation élevé.

L'unique souci des familles en France est celui de préserver l'identité et les valeurs d'origine par le mariage avec quelqu'un du pays. Les parents expliquent souvent d'ailleurs qu'ils souhaitent ajouter du "sang frais". Ils estiment que leurs enfants nés en France perdent une bonne partie de leur culture turque et que l'arrivée d'un conjoint de Turquie leur permettra d'avoir des petits-enfants qui, eux, seront "turcs". 


\section{S'il y a une prise en compte de la "francisation" des enfants, l'action des familles vise-t-elle à contrebalancer cette évolution ?}

Effectivement. Mais cette évolution répond aussi à un autre enjeu familial. Souvent les frères et sceurs se promettent mutuellement de marier ensemble leurs enfants, afin que les enfants des proches restés au pays puissent venir en France. C'est donc aussi parfois une stratégie familiale visant à faire immigrer quelqu'un de la famille.

\section{Rencontre-t-on la situation inverse, c'est-à-dire des personnes envoyées en Turquie pour y être mariées, et qui vont rester?}

Ces cas sont très rares, souvent pratiqués en guise de "punition" pour les filles. En même temps, la famille peut souhaiter marier son enfant à quelqu'un ayant une très belle position économique et sociale. Dans ce cas, il s'agit plutôt d'un choix du couple par rapport à l'avenir.

\section{Quels sont les autres problèmes de transmission au sein des familles turques?}

La première génération d'immigrés turcs ne parle pas le français, alors que la deuxième génération le parle bien, mais s'exprime dans un turc médiocre, essentiellement parlé et appris auprès des parents, issus des villages et peu éduqués. Les parents ne peuvent donc pas transmettre la grammaire ou la structure de la langue à leurs enfants, qui apprennent donc un turc très basique et avec un fort accent dialectal.

Lorsque la deuxième génération fait venir son conjoint de Turquie, les nouveaux couples ne laissent pas leurs enfants dans les crèches, mais les font garder par les grand-mères. Comme souvent elles ne parlent pas le français, les enfants grandissent en entendant un turc médiocre et arrivent en primaire en ne parlant pas un mot de français. Ce qui est incompréhensible pour les maîtres, qui rencontrent pourtant des parents complètement francophones, ou au moins l'un des deux.

L'identité se transmet avec l'histoire de la famille; celle de l'immigration aussi. Le fait central est d'avoir posé ses bagages quelque part, ou bien de ne pas les poser. Le discours qui caractérisait l'immigration turque au départ était le suivant : "Je reste un peu et je repartirai." Il n'y avait donc pas d'investissement dans le pays d'accueil, et cela a fini par faire naître quelque chose d'instable auprès de la deuxième génération. Celle-ci a peut-être toujours entendu la même chose dans la famille : 
"On ne va pas rester. Ici, ce n'est pas chez nous." Qu'est-ce que les familles ont transmis pour que les générations suivantes puissent finalement se positionner dans la société française? C'est là une sacrée question!

\section{N'y a-t-il pas de plus en plus de migrants turcs qui développent au contraire une stratégie qui leur permette d'être à la fois ici et là-bas ?}

Certains jeunes ont effectivement une identité double. Ils sont à l'aise dans les deux sociétés, comme c'est le cas des enfants binationaux, à qui on ne demande jamais : "Tu es qui ? Tu es Turc ou Français?' Ces enfants vivent très bien dans les deux pays, circulent de l'un à l'autre. Ce sont des transnationaux. Ils ont adopté le principe suivant: "Je suis ici, mais je pourrais aussi bien être dans mon pays d'origine, ou dans un autre pays ailleurs en Europe."

Il n'y a pas eu d'études sur le sujet, qui permettraient d'avoir des chiffres précis, mais d'après ce que nous avons pu constater, il y a de plus en plus de jeunes qui ont des métiers un peu "passe-partout", qui font des projets dans une perspective liée au pays d'origine ou à d'autres pays en Europe. Dans ce sens-là, il y a aussi un certain dynamisme.

Cela n'empêche pas ces jeunes de se considérer comme installés en France, mais ils sont capables d'être mobiles. Même s'ils achètent une maison, il se peut qu'ils partent dans un autre département où le secteur du travail est plus ouvert. Il est vrai que la spécificité des Turcs a toujours été de ne pas jeter l'ancre définitivement quelque part. L'achat d'une maison veut certes dire que l'on s'installe, mais c'est aussi un moyen de s'éloigner des autres. De même, la création d'une entreprise répond à cette même logique : les Turcs préfèrent créer leurs entreprises pour pouvoir embaucher des Turcs, car ils les connaissent mieux et peuvent mieux les gérer. Il y a une situation de confiance, mais aussi des enjeux de pouvoir. Ce sont donc à la fois des indicateurs de l'intégration, mais aussi des freins par une attitude qui consiste à se démarquer.

\section{Observez-vous des situations de cohabitation entre les familles turques qui résident dans le même immeuble ou dans le même quartier?}

Oui, cela existe. À Plaisir dans les Yvelines, par exemple, un groupe de mamans s'est créé et je n'ai jamais vu autant de mixité. Les familles se mélangent et cohabitent très facilement. En effet, il n'y a pas d'association turque, parce que les Turcs ne sont pas nombreux et qu'ils ne viennent pas du même coin de la Turquie. Ce vide communautaire permet aux personnes d'être plus ouvertes vers la société dans son ensemble. 
Comment les familles turques qui vivent depuis plusieurs décennies en France perçoivent-elles la société et les familles françaises ? Est-ce qu'il y a un discours mettant en avant des situations de discrimination?

Non, peut-être parce que les enfants ont grandi et n'hésitent pas à parler de discrimination, alors qu'au début les parents ne savaient pas que telle pratique à leur égard était une discrimination, faute de ne pas connaître cette problématique. Les Turcs n'ont généralement pas de difficultés avec les Français. Ils cohabitent cordialement, mais sans se connaître vraiment. Cependant, le "monde français" ne doit pas rentrer dans leur famille. Ils accueillent volontiers occasionnellement les voisins "français" à leur domicile, très heureux de leur faire découvrir d'où ils viennent, mais ils visitent les familles françaises extrêmement rarement.

Comme les Turcs ne sont pas dans une relation intense avec la société et les familles françaises, ils n'ont pas forcément rencontré de situations discriminatoires. Pour pouvoir dire "je suis discriminê", il faut être continuellement en lien avec la société, dans le dialogue, dans la relation. C'est cela qui est à la base de l'inclusion ou de l'exclusion.

\section{Pensez-vous donc que cette situation provienne de la discrétion de l'immigration turque, ou bien du fait que la société française ne s'in- téresse pas à ces migrants ?}

Lorsque nous rencontrons les associations turques, pour parler d'intégration par exemple, leurs membres nous disent : "Quand nous sommes arrivés ici, on travaillait bien, personne ne nous disait rien, personne ne nous a demandé si on connaissait la langue ou a fait l'effort de nous apprendre le français. Aujourd'hui, quarante ans après, on nous demande pourquoi nous vivons comme cela, pourquoi nous ne sommes pas intégrés. C'est un peu tard!' Il y a effectivement eu un écueil à un moment donné.

\section{Vous parliez de la déception des jeunes mariés lors de leur arrivée en France. Quelle est la situation des couples issus d'un mariage arrangé ?}

Il y a également un problème de communication au sein du couple. Quelqu'un qui apprend bien sa langue maternelle n'aura pas de problèmes pour acquérir le français : être bilingue n'a jamais été un problème. Pourtant, le fait qu'un jeune né en France, qui maîtrise mal la langue turque, se trouve face à un conjoint turc, qui maîtrise bien la langue turque et mal le français, constitue une réelle difficulté, 
qui s'ajoute aux difficultés qui existent déjà dans le cadre d'un mariage arrangé. Le conjoint qui vient de Turquie se trouve aussi dans un contexte nouveau, la communication manque et la structure familiale est différente dans les familles installées ici. Par exemple, les enfants, et notamment les garçons, restent attachés aux parents plus longtemps.

Notre hypothèse est que cette nouvelle génération venant de Turquie va pouvoir être le vecteur de l'évolution des familles installées ici, du moins pour ceux qui ont le courage d'aller jusqu'au bout. Il y a autant d'hommes que de femmes qui trouvent la situation en France dépassée et ont envie d'aller plus loin. Après, tout dépend du lien avec la communauté dans laquelle ils se trouvent. Est-ce qu'elle va les laisser faire ? Est-ce qu'ils vont être autonomes pour aller de l'avant vis-à-vis de leurs familles et de leur communauté ?

\section{Quelles contraintes peuvent exercer les familles sur les conjoints ayant des velléités d'indépendance?}

Pendant au moins cinq ans, tous ces conjoints, y compris les hommes, sont soumis à une sorte de chantage de la part de la famille. Si, par exemple, le conjoint souhaite exercer un métier différent de celui qui lui est proposé dans l'entreprise du beau-père, ce dernier peut le menacer d'annuler sa carte de séjour. Il faut rappeler que les jeunes travaillent souvent avec les beaux-parents, et, pour la plupart, sans recevoir de salaire en échange.

Sur un plan plus personnel, les jeunes hommes se plaignent également de ne pas pouvoir manger en tête-à-tête avec leur conjointe, ou encore de ne pas pouvoir marcher dans la rue main dans la main avec elle, car cela n'est pas toléré ici par les familles.

\section{La situation de couple est donc compliquée à vivre dans ces familles ?}

Prenons l'exemple d'un jeune couple qui a choisi de se marier. Ils sont mariés, c'est vrai, ils ont signé le livret de famille, ils ont fait la fête, mais ils n'existent pas en tant qu'entité "couple" : ils sont une entité "famille". Or les conjoints pourraient mieux se connaitre et bien s'entendre si on leur laissait la possibilité de se retrouver seuls. Mais, de part et d'autre, les familles élargies - parents, frères et sceurs, cousins, etc. - vont tellement s'immiscer dans leur vie qu'il ne s'agira plus de leur propre vie, mais d'une vie familiale, globale. Nous entendons fréquemment chez les jeunes gens des propos du type: "Je ne peux pas me rendre au café seul avec ma femme, car si je le fais, je vais avoir des problèmes." En 2009, c'est assez dramatique d'avoir à entendre cela. 


\section{Est-ce que ces mêmes problèmes se posent dans les villages d'ori- gine ?}

Non, ou plutôt oui, mais d'une autre manière. Dans les villes, la société a évolué, certaines normes ou certains repères ont éclaté et d'autres se sont mis en place. On peut bien sûr se promener en tant que couple. Les manifestations de tendresse en public restent proscrites par la tradition, mais il y a d'autres moyens pour deux amoureux de se retrouver, parfois avec la complicité des tantes.

\section{La femme est-elle enfermée dans son rôle de femme au foyer?}

Lorsqu'un conflit intergénérationnel éclate, il arrive qu'on s'entende mieux avec le père qu'avec la mère. En effet, les pères ont généralement un contact avec le monde à l'extérieur de la famille, avec d'autres personnes. Ils ont pu intégrer un certain nombre de normes et de valeurs. Au contraire, les femmes, qui restent le plus souvent à l'intérieur de la maison, qui n'ont pas toujours quelque chose à transmettre à leurs enfants, imposent des règles absurdes. Parfois, on se demande où elles trouvent ces règles qui ne relèvent ni de la religion ni de la tradition.

La femme est gardienne de la tradition, et le fait qu'elle n'ait pas de contact avec d'autres personnes extérieures à la communauté est problématique. Les femmes ne se retrouvent qu'avec des femmes qui vivent la même situation, qui ont la même vision des choses. Lorsque l'une d'entre elles se distingue par des propos qui marquent une certaine ouverture, une certaine rupture, elle est de toute façon exclue du groupe.

Apparemment, ce serait un souhait émanant de l'État turc, afin qu'il y ait dans chaque association turque une branche féminine, mais nous craignons que cette initiative soit davantage prise pour transmettre des valeurs traditionnelles que pour autre chose.

Cela dit, l'équipe de notre association, majoritairement féminine, se présente tout de même déjà comme un véritable rouage à l'intérieur d'un mécanisme de changement. Les hommes turcs ne semblent pas avoir de problèmes à être en contact avec des travailleuses sociales.

\section{Est-ce que la religion est un ferment familial ? Est-ce qu'il y a un enseignement religieux dispensé en famille?}

Oui, et même les familles qui ne sont pas pratiquantes envoient leurs enfants assister aux cours sur le Coran. L'idée étant que, de toutes les façons, il faut que l'enfant 
sache, afin qu'il puisse pratiquer ensuite sa religion. Il y a une incitation à savoir, mais pas à comprendre. Dans le meilleur des cas, l'enfant sera envoyé pour participer à cet apprentissage religieux, même s'il n'est pas dispensé au sein des familles. Ceci même chez les familles ouvertes.

Ce retour au religieux se fait aussi à l'échelle nationale. Dans certains quartiers ou certaines villes, les femmes turques, maghrébines ou pakistanaises sortent voilées. Les femmes turques, pour certaines d'entre elles, ne souhaitent pas porter le voile et sont pourtant obligées de le faire. Il y a même des femmes dont le mari ne souhaite pas qu'elles portent le voile, mais qui le font, soit parce qu'elles en avaient l'habitude dans leur village en Turquie, soit parce qu'elles le voient porter autour d'elles.

On peut citer l'exemple de cette femme accueillie à l'aéroport en France par son mari. En arrivant chez eux, celui-ci s'est arrêté pour lui donner un voile : "Désormais, tu mettras cela, on est en France!' Nombre de filles qui n'ont jamais porté de foulard de leur vie en Turquie le portent en France.

\section{À propos des violences conjugales, comment s'en sortent ces fem- mes ? Arrivent-elles à rester dans la famille ? Faut-il envisager une séparation?}

Le problème vient souvent du fait qu'elles n'arrivent pas à trouver les bons interlocuteurs. Les situations que nous rencontrons à ELELE ne représentent qu'une minorité par rapport à la réalité. Lorsqu'elle s'est décidée, la femme qui veut partir de chez elle ne fera pas marche arrière, mais c'est une éventualité très rare. Le problème de ces femmes est de trouver quelqu'un à qui confier sa détresse et de pouvoir être accompagnées pour savoir quoi faire.

Pour beaucoup, entamer une procédure de divorce est une faute. Les femmes voient la violence comme une normalité lorsqu'elles ont été élevées d'une certaine manière. Une fille est éduquée pour être la future femme d'Untel. D'ailleurs, on dit pour une fille : "El kapisina, gider", c'est-à-dire "elle ira à la porte de quelqu'un d'autre", au service de la belle-famille.

De plus, la femme doit être garante de l'unité familiale, même si le mari est le fautif. Les femmes qui arrivent à s'exprimer reçoivent des éléments d'informations pour prendre leur décision de manière autonome. Le temps de maturation peut être long. Des lieux comme ELELE manquent. Pour une femme qui ne parle pas bien le français, la langue reste une barrière, et les services sociaux n'essayeront pas forcément de comprendre le cadre familial qui est à l'origine du sentiment de culpabilité qu'elle ressent de devoir quitter son monde. Les jeunes femmes portent encore le lourd fardeau des normes traditionnelles. 


\section{Les violences sont-elles plus fréquentes dans les premières années ?}

Les situations sont très variées. La violence vient aussi du fait que ces jeunes femmes résistent à la situation qui leur est imposée. Elles n'acceptent pas d'être des "esclaves". C'est vrai pour la violence physique, mais il y a toute une violence psychologique, morale et financière qui est tout aussi difficile à gérer.

On peut rencontrer dans le même après-midi une jeune femme de vingt ans tout juste arrivée de Turquie et victime de violences, tout comme une femme proche de la cinquantaine, installée en France depuis plus de trente ans ! De plus, beaucoup de femmes primo-arrivantes acceptent de subir, par peur de perdre leur carte de séjour, étant données les règles légales actuelles. La situation est pire pour celles qui sont arrivées clandestinement, ou avec des faux papiers d'identité. Cette pression de l'inégalité s'ajoute aux mensonges, aux non-dits et au déni de l'identité de l'individu. Toute violence morale est de toute façon difficile à prouver, et un assistant social peut très bien dire: "Écoutez madame, si vous le souhaitez, vous pouvez vous séparer, c'est votre droit. Par contre, sachez que dans ce cas votre carte de séjour ne sera pas renouvelée." Malgré les preuves de violence fournies, dans certains départements, de nombreuses femmes sont renvoyées en Turquie. Elles sont donc doublement punies.

C'est un chantage qui pèse également sur les hommes. Récemment, il y a eu le cas d'un jeune homme qui est parti pour ne plus avoir à subir d'humiliations et qui, une fois en Turquie, a entamé une procédure légale contre sa belle-famille en France, l'accusant d'avoir organisé son mariage avec une femme qui n'était pas consentante. Hommes et femmes réagissent bien sûr de manières différentes, mais les conséquences peuvent être beaucoup plus lourdes pour les femmes, qui sont souvent reconnues "coupables" par leur propre famille.

Il faut informer ces femmes, leur expliquer que tous ces actes de harcèlement sont des crimes. Il y a trois ans, nous avons discuté de cette problématique avec une délégation du statut de la femme, l'équivalent du service des droits des femmes du gouvernement turc, et cette année ils ont édité une plaquette que nous avons rédigé ensemble, qui explique les différentes violences dont les femmes turques de France sont les victimes, avec des adresses utiles en cas de problème.

\section{Est-ce qu'il y a des comparaisons à faire entre les différents pays d'installation des Turcs ? Quelle est par exemple la situation des Turcs en Allemagne ? Est-elle plus enviable pour les immigrés turcs?}

La France n'est pas un pays forcément très raciste. Les Turcs d'Allemagne trouvent qu'il y a un sentiment discriminatoire plus prononcé outre-Rhin qu'en 
France. Les Turcs d'Allemagne trouvent aussi que la société allemande est beaucoup plus organisée que la France, et que la société française ressemble davantage à leur société. Ce qui fait qu'ils disent trouver un peu de leur pays en France. En revanche, les Turcs de France trouvent que les Turcs d'Allemagne vivent mieux, ont de meilleures chances de réussite socio-économique, malgré la politique allemande qui les a bousculés. En raison des discriminations, les Turcs ont été beaucoup plus revendicatifs, ont voulu trouver leur place, montrer qu'ils pouvaient réussir.

De plus, les autorités allemandes ont choisi des Turcs ayant de bonnes qualifications, alors qu'on trouve ici en France beaucoup de personnes moins qualifiées, issues des villages. Ce sont des Turcs qui envisageaient d'aller en Allemagne, mais qui se sont finalement arrêtés en Savoie, ou en Alsace, car il y avait du travail, et non parce qu'ils ont été refoulés à la frontière allemande. Pendant longtemps, on a dit que la France avait "ramassé" les Turcs qui avaient été refusés en Allemagne. Or des études ont prouvé que ce n'était pas vrai, mais, par contre, on sait que beaucoup d'immigrés turcs de France de première génération auraient préféré, au départ, se retrouver en Allemagne. 